Military Technical College Kobry El-Kobbah, Cairo, Egypt

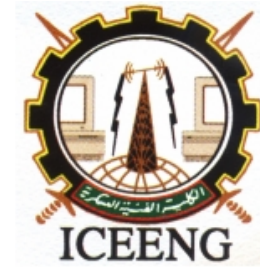

\author{
$8^{\text {th }}$ International Conference \\ on Electrical Engineering \\ ICEENG 2012
}

\title{
A Comparison Study between Inferred State-Space and Neural Network Based System Identifications Using Adaptive Genetic Algorithm for Unmanned Helicopter Model
}

\author{
By
}

Ahmed M. Hosny*

\section{Abstract:}

In this paper, system identifications of an unmanned aerial vehicle (UAV) based on inferred state space and multiple neural networks were presented. In this work an optimization approach was used to conclude an inferred state space and the multiple neural networks system identifications based on the genetic algorithms separately. The UAV is a multi-input multi-output (MIMO) nonlinear system. Models for such MIMO system are expected to be adaptive to dynamic behavior and robust to environmental variations. This task of accurate modeling has been achieved with multi-neural network architecture in the most recent years. The presented work is focusing on an inferred state space based system identification which is a new approach seldom used, but it is also easier and more stable compared with the multi-network based system identification during the modeling of dynamic behavior of nonlinear systems. In other words the number of inputs used in the genetic algorithm to obtain an inferred state space is almost one third of the number of inputs needed to develop the multi-layer recurrent neural network architecture to simulate the required dynamic behavior of a real model. The neural network models are based on the autoregressive technique with linear and nonlinear networks. The simulation results presented in this paper show the superiority of the inferred state space model compared with the autoregressive technique based multi-neural network.

\section{Keywords:}

UAV Unmanned Aerial Vehicle, RC Remote Control, PI Performance Index, RNN Recurrent Neural Network, GA Genetic Algorithm, ISS Inferred State Space.

* Egyptian Armed Forces 


\section{Helicopter Model}

Helicopter dynamics obey the Newton-Euler equations for rigid body in translational and rotational motions. The helicopter dynamics can be studied by employing lumped parameter approach which presents that the helicopter model shown in Figure (1) as a composition of following components; main rotor, tail rotor, fuselage, horizontal bar and vertical bar. Figure (2) illustrates typical arrangement of component forces and moments generated in helicopter simulation model, Table (1) illustrates Raptor 90 helicopter model specifications [1,2].

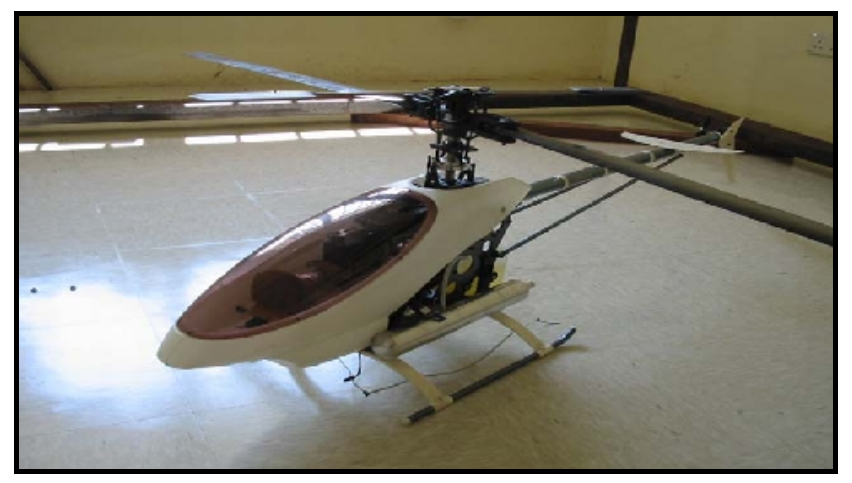

Figure (1): Raptor 90 (15 cc Engine)

Forces equations:

$\dot{u}=-(w q-v r)+\frac{X}{m}-g \sin \theta$

$\dot{v}=-(u r-w p)+\frac{Y}{m}+g \cos \theta \sin \varphi$

$\dot{w}=-(v p-u q)+\frac{Z}{m}+g \cos \theta \cos \varphi$

Moment equations:

$$
\begin{aligned}
& I_{x x} \dot{p}=\left(I_{y y}-I_{z z}\right) q r+I_{x z}(\dot{r}+p q)+L \\
& I_{y y} \dot{q}=\left(I_{z z}-I_{x x}\right) r p+I_{x z}\left(r^{2}-p^{2}\right)+M \\
& I_{z z} \dot{r}=\left(I_{x x}-I_{y y}\right) p q+I_{x z}(\dot{p}-q r)+N
\end{aligned}
$$

Kinematic equations:

$$
\begin{aligned}
& \dot{\varphi}=p+q \sin \varphi \tan \theta+r \cos \varphi \tan \theta \\
& \dot{\theta}=q \cos \varphi-r \sin \varphi \\
& \dot{\Psi}=q \sin \varphi \sec \theta+r \cos \varphi \sec \theta
\end{aligned}
$$


Table (1): Parameters of Raptor 90 helicopter for simulation model

\begin{tabular}{|c|c|}
\hline Parameter & Description \\
\hline$\rho=1.225 \mathrm{~kg} / \mathrm{m}^{3}$ & Atmosphere density \\
\hline $\mathrm{m}=7.70 \mathrm{~kg}$ & Helicopter mass \\
\hline$I_{x x}=0.192 \mathrm{~kg} \mathrm{~m}^{2}$ & Rolling moment of inertia \\
\hline$I_{y y}=0.34 \mathrm{~kg} \mathrm{~m}^{2}$ & Pitching moment of inertia \\
\hline$I_{z z}=0.280 \mathrm{~kg} \mathrm{~m}^{2}$ & Yawing moment of inertia \\
\hline nom $=162 \mathrm{rad} / \mathrm{s}$ & Nominal main rotor speed \\
\hline $\mathbf{R}_{\mathrm{M}}=0.775 \mathrm{~m}$ & ain rotor radius \\
\hline$R_{\mathrm{CR}}=0.370 \mathrm{~m}$ & Stabilizer bar radius \\
\hline $\mathrm{C}_{\mathrm{M}}=0.058 \mathrm{~m}$ & Main rotor chord \\
\hline $\mathrm{C}_{\mathrm{CR}}=0.06 \mathrm{~m}$ & Stabilizer bar chord \\
\hline $\mathrm{a}_{\mathrm{M}}=5.5 \mathrm{rad}^{-1}$ & Main rotor blade lift curve slope \\
\hline$C_{D d}^{M}=\mathbf{0 . 0 2 4}$ & Main rotor blade zero lift drag coefficient \\
\hline$C_{T_{\max }}^{\mathrm{N}}=\mathbf{0 . 0 0 1 6 8}$ & Main rotor max thrust coefficient \\
\hline$I_{\beta}=0.038 \mathrm{~kg} \mathrm{~m}^{2}$ & Main rotor blade flapping inertia \\
\hline$R_{T}=0.13 \mathrm{~m}$ & Tail rotor radius \\
\hline $\mathrm{C}_{\mathrm{T}}=0.029 \mathrm{~m}$ & Tail rotor chord \\
\hline$a_{\mathrm{T}}=5.0 \mathrm{rad}^{-1}$ & Tail rotor blade lift curve slope \\
\hline$C_{D_{0}}^{T}=0.024$ & Tail rotor blade zero lift drag coefficient \\
\hline$C_{T_{\max }}^{T}=\mathbf{0 . 0 9 2 2}$ & ail rotor max thrust coefficient \\
\hline$n_{T}=4.66$ & Gear ratio of tail rotor to main rotor \\
\hline $\mathbf{n}_{\mathrm{es}}=\mathbf{9 . 0}$ & Gear ratio of engine shaft to main rotor \\
\hline$\delta_{\tau}^{\text {Grim }}=0.1 \mathrm{rad}$ & Tail rotor pitch trim offset \\
\hline $\mathrm{S}_{\mathrm{V}}=0.012 \mathrm{~m}^{2}$ & Effective vertical fin area \\
\hline $\mathrm{S}_{\mathrm{H}}=0.01 \mathrm{~m}^{2}$ & Effective horizontal fin area \\
\hline$C_{L x}^{V}=2.0 \mathrm{rad}^{-1}$ & Vertical fin lift curve slope \\
\hline$C_{L x}^{R}=3.0 \mathrm{rad}^{-1}$ & Horizontal tail lift curve slope \\
\hline$s_{x}^{F}=0.1 \mathrm{~m}^{2}$ & Frontal fuselage drag area \\
\hline$s_{y}^{F}=\mathbf{0 . 2 2} \mathrm{m}^{2}$ & Side fuselage drag area \\
\hline$s_{z}^{f}=0.15 \mathrm{~m}^{2}$ & Vertical fuselage drag area \\
\hline $\mathbf{h}_{\mathrm{M}}=\mathbf{0 . 2 3 5 \mathrm { m }}$ & Main rotor hub height above CG \\
\hline $\mathrm{l}_{\mathrm{M}}=\mathbf{0 . 0 1 5 \mathrm { m }}$ & Main rotor hub behind CG \\
\hline $\mathrm{l}_{\mathrm{T}}=0.91 \mathrm{~m}$ & Tail rotor hub location behind CG \\
\hline $\mathbf{h}_{\mathrm{T}}=\mathbf{0 . 0 8 \mathrm { m }}$ & Tail rotor height above CG \\
\hline$l_{H}=0.71 \mathrm{~m}$ & Stabilizer location behind CG \\
\hline $\mathrm{k}_{\mathrm{MR}}=\mathbf{0 . 3 3 3 3}$ & Amount of commanded swash plate tilt \\
\hline $\mathrm{k}_{\mathrm{CR}}=\mathbf{1 . 1 4 2 9}$ & Geometry coefficient of the mechanical linkage of control rotor and swash plate \\
\hline
\end{tabular}




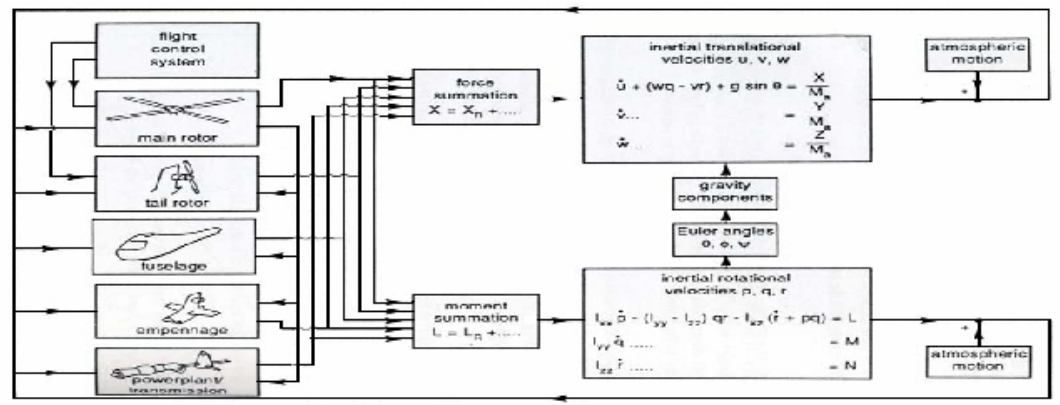

Figure (2): Typical Arrangement of Component Forces and Moments Generation in 6-DOF Helicopter Simulation Model

The linearized matrices for hover condition (or low speed flight) excluding the control rotor (Six DOF model eight states) are given in Table (2) and Table (3). Each column and row is marked with the states and inputs that are being referred to the state space model. Table (4) states the stable and unstable eigenvalues according to the different modes for six DOF in hovering and low speed flight conditions. Figure (3) illustrates the different poles on the corresponding Pole-Zero Map.

$$
\begin{gathered}
\dot{x}=A x+B u \\
y=C x
\end{gathered}
$$

Table (2): Analytically obtained A matrix in hover with no control rotor

\begin{tabular}{|c|c|c|c|c|c|c|c|c|}
\hline \hline & $u$ & $w$ & $q$ & $\theta$ & $v$ & $p$ & $\varphi$ & $r$ \\
\hline$u$ & -0.0070825 & 0 & 0.00093895 & -9.81 & -0.0292 & -0.0292 & 0 & 0 \\
\hline$w$ & 0 & -0.8159 & 0 & 0 & 0 & 0 & 0 & -0.12 \\
\hline$q$ & 0.0377 & -0.2775 & -0.6718 & 0 & -0.28599 & 0.1558 & 0 & 0.265 \\
\hline$\theta$ & 0 & 0 & 1 & 0 & 0 & 0 & 0 & 0 \\
\hline$v$ & 0.00093895 & 0 & 0.0144 & 0 & -0.06808 & 0.122823 & 9.81 & 0.055 \\
\hline$p$ & 0.0094513 & 0 & -0.2942 & 0 & -0.1377 & -1.286 & 0 & 0.19 \\
\hline$\varphi$ & 0 & 0 & 0 & 0 & 0 & 1 & 0 & 0 \\
\hline$r$ & 0 & -1.5246 & 0 & 0 & 1.528 & 0.122 & 0 & -5.1868 \\
\hline \hline
\end{tabular}

Table (3): Analytically obtained $\boldsymbol{B}$ matrix in hover with no control rotor

\begin{tabular}{|c|c|c|c|c|}
\hline \hline & $\delta_{\text {Col }}$ & $\delta_{\text {Lon }}$ & $\delta_{\text {Lat }}$ & $\delta_{\text {Ped }}$ \\
\hline$u$ & 5.2981 & 1.5591 & -0.1816 & 0 \\
\hline$w$ & -128.777 & 0 & 0 & 0 \\
\hline$q$ & -72.0367 & -8.3082 & 0.9678 & 9.07 \\
\hline$\theta$ & 0 & 0 & 0 & 0 \\
\hline$v$ & -31.9088 & 0.0605 & -0.5196 & 5.055 \\
\hline$p$ & -321.1883 & 1.8281 & -15.6933 & 17.322 \\
\hline$\varphi$ & 0 & 0 & 0 & 0 \\
\hline$r$ & 178.2831 & 0 & 0 & 17.322 \\
\hline
\end{tabular}


Table (4): Eigenvalues and modes for 6-DOF in Hovering/Low Speed Flight Condition

\begin{tabular}{|c|c|c|c|}
\hline \hline Mode & Eigenvalues & Damping & Frequency (rad/s) \\
\hline Longitudinal Oscillation & $0.169 \pm 0.392 \mathbf{i}$ & -0.395 & 0.427 \\
\hline Lateral Oscillation & $-0.0279 \pm 0.765 \mathbf{i}$ & 0.0365 & 0.765 \\
\hline Heave & $-0.68 \pm 0.142 \mathbf{i}$ & 0.979 & 0.695 \\
\hline Roll Subsidence & -1.68 & 1 & 1.68 \\
\hline Yaw & -5.28 & $\mathbf{1}$ & 5.28 \\
\hline \hline
\end{tabular}

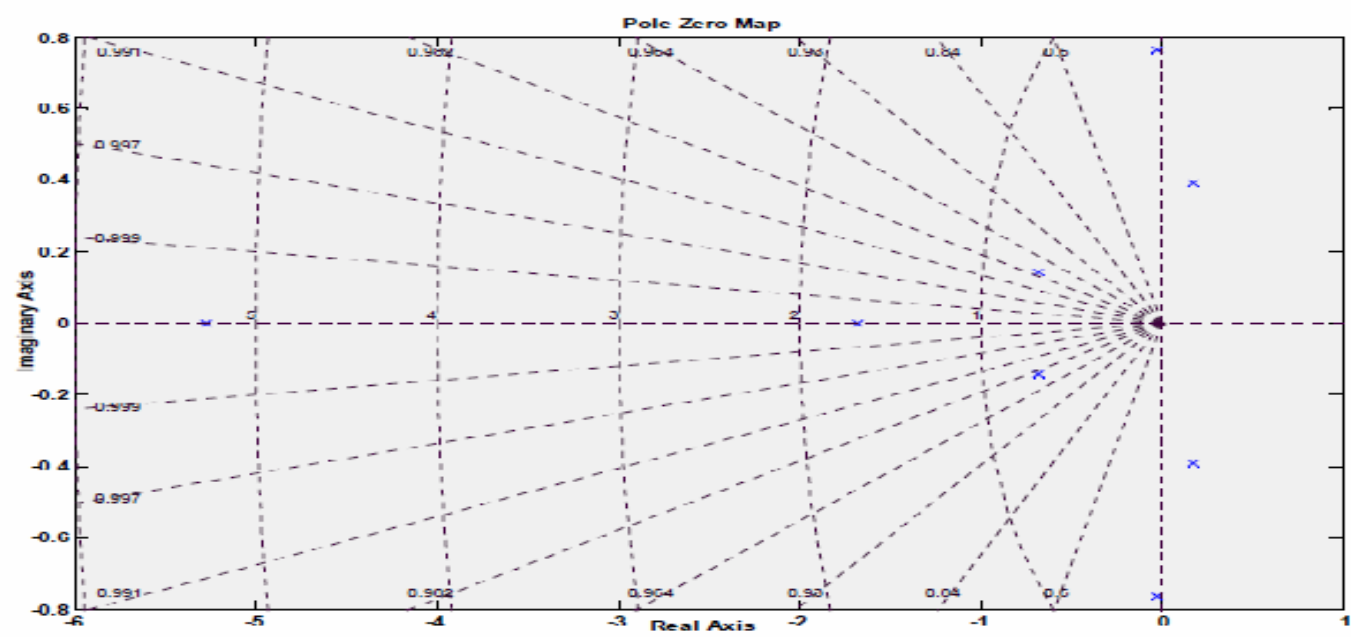

Figure (3): Poles of Coupled Longitudinal and Lateral Motion for 6-DOF with no Control Rotor

The algebraic state space matrices shown in Figure (4) are usually used to calculate all the matrices elements that require all the aerodynamic coefficients and stability derivative values. This tedious job will acquire a considerable number of hours though the final error sometimes exceeds the acceptable limitations. The final error is usually due to many reasons such as (nonlinearities, approximations, uncertainties and miscalculations) therefore to build up a practical model (state space model) it is recommended to apply some methodology based on practical experiments results to extract all the required state space elements. 


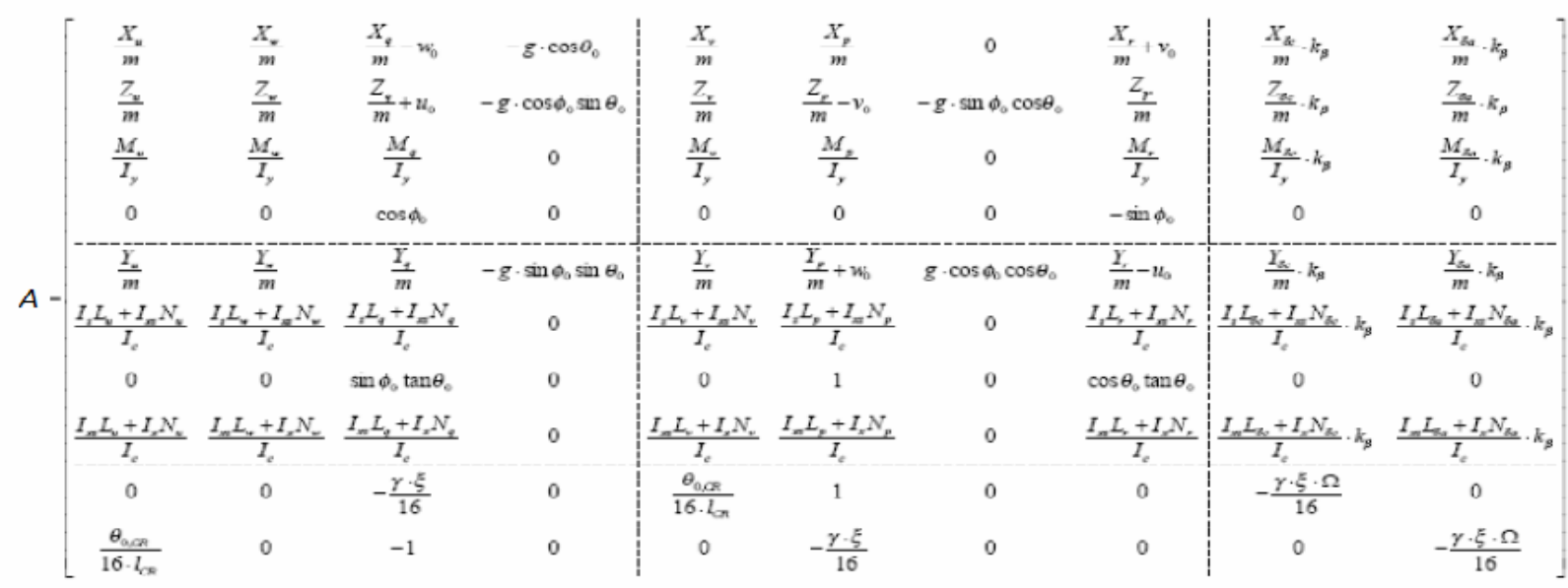

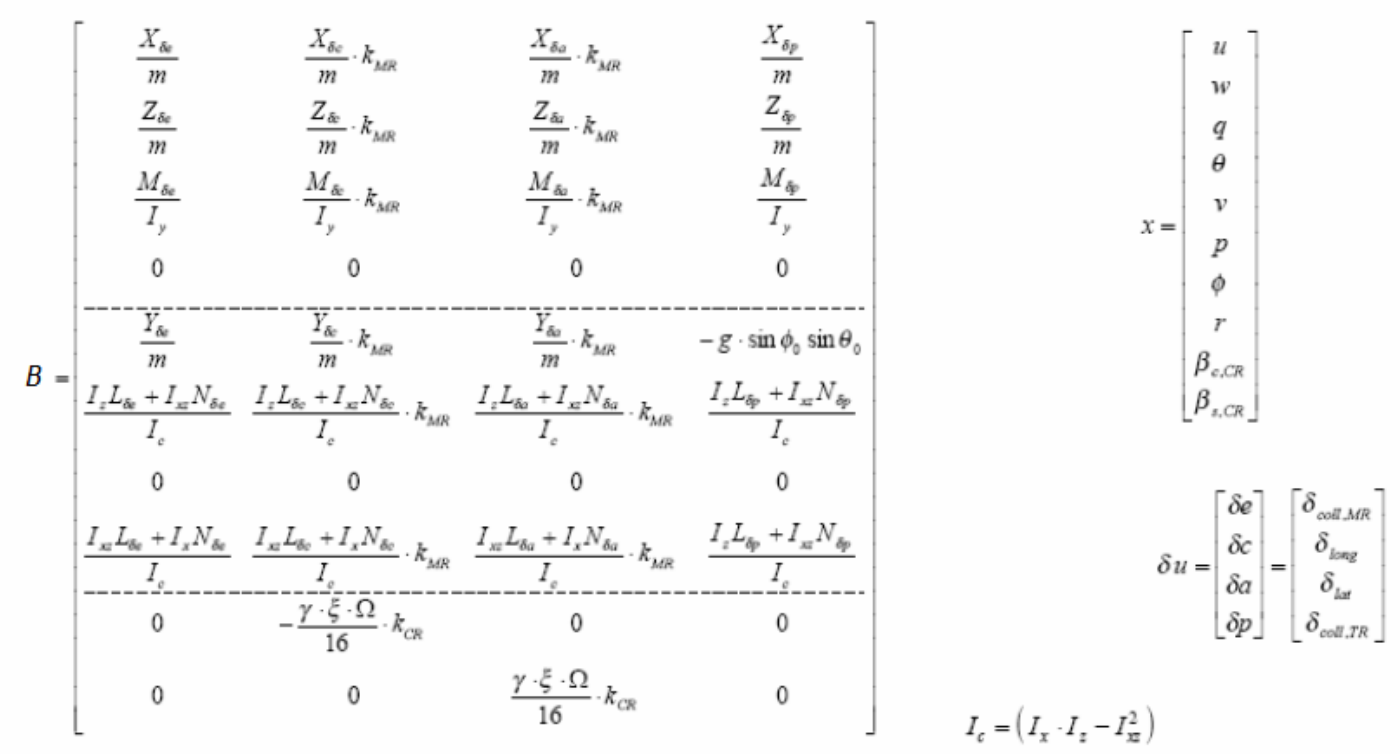

$\delta_{c o i, M R}=\theta_{0}$ Commanded main rotor collective angle

$\delta_{\text {long }}=B_{1, S P}$ Longitudinal swashplate tilt

$\delta_{\text {lat }}=A_{1, S P}$ Lateral swashplate tilt

$\delta_{\text {col }, T R}=\theta_{O T}$ Commanded tail rotor collective angle

Figure (4): Algebraic State Space Matrices

\section{Methodology of Inferring State Space Elements}

It is not realistic to calculate all the state space elements analytically but it is recommended to use these algebraic state space matrices only to roughly determine the range of each element to ease the GA process to obtain proper solutions in less time. Some of the state space elements are already known that would reduce the number of 
the total elements. The unknown elements ranges only should be roughly determined in order to decrease the number of iterations needed to obtain an acceptable solution that represents the most global minimum of the performance index (the minimum error between the inferred and real state space values). Table 5 and Table 6 show the known and unknown elements in matrix A and matrix B. After calculating the state space elements ranges by using the proposed algebraic state space or by the analogy of some similar model GA is applied to calculate the inferred state space elements as the following procedure.

\subsection{Using binary system coding}

Coding $\square$ using 10-bit binary genes to express $\left(A_{1}, A_{2}, \ldots \ldots \ldots A_{26}\right)$ and $\left(\mathrm{B}_{1}\right.$, $\left.\mathrm{B}_{2}, \ldots \ldots \ldots \mathrm{B}_{18}\right)$. For example $\left(A_{l}\right)$ from $0000000000(0)$, to is 1111111111(1023).Then string $A_{2} \ldots$ etc. till $B_{18}$ to 440-bit binary cluster. $x: 0000110111 \ldots \ldots \ldots . .11011100010000100010$ expresses a chromosome $\square$ the former 10-bit express $\left(A_{1}\right)$, the second portion express $\left(A_{2}\right)$ and the third one express the $\left(A_{3}\right) \ldots$ etc.

Decoding $\square$ Cut one string of 440-bit binary string to 44 10-bit binary string $\square$ then converts them to decimal system values $(A 1, A 2, \ldots \ldots \ldots A 26)$ and $(B 1, B 2, \ldots \ldots \ldots B 18)$, $[3,4,5,6]$.

Table (5): Analytically obtained A matrix in hover with no control rotor

\begin{tabular}{|c|c|c|c|c|c|c|c|c|}
\hline \hline & $u$ & $w$ & $q$ & $\theta$ & $v$ & $p$ & $\varphi$ & $r$ \\
\hline$u$ & $\mathrm{~A} 1$ & 0 & $\mathrm{~A} 2$ & -9.81 & $\mathrm{~A} 3$ & $\mathrm{~A} 4$ & 0 & 0 \\
\hline$w$ & 0 & $\mathrm{~A} 5$ & 0 & 0 & 0 & 0 & 0 & $\mathrm{~A} 6$ \\
\hline$q$ & $\mathrm{~A} 7$ & $\mathrm{~A} 8$ & $\mathrm{~A} 9$ & 0 & $\mathrm{~A} 10$ & $\mathrm{~A} 11$ & 0 & $\mathrm{~A} 12$ \\
\hline$\theta$ & 0 & 0 & 1 & 0 & 0 & 0 & 0 & 0 \\
\hline$v$ & $\mathrm{~A} 13$ & 0 & $\mathrm{~A} 14$ & 0 & $\mathrm{~A} 15$ & $\mathrm{~A} 16$ & 9.81 & $\mathrm{~A} 17$ \\
\hline$p$ & $\mathrm{~A} 18$ & 0 & $\mathrm{~A} 19$ & 0 & $\mathrm{~A} 20$ & $\mathrm{~A} 21$ & 0 & $\mathrm{~A} 22$ \\
\hline$\varphi$ & 0 & 0 & 0 & 0 & 0 & 1 & 0 & 0 \\
\hline$r$ & 0 & $\mathrm{~A} 23$ & 0 & 0 & $\mathrm{~A} 24$ & $\mathrm{~A} 25$ & 0 & $\mathrm{~A} 26$ \\
\hline
\end{tabular}

Table (6): Analytically obtained $\boldsymbol{B}$ matrix in hover with no control rotor

\begin{tabular}{|c|c|c|c|c|}
\hline \hline & $\delta_{\text {Col }}$ & $\delta_{\text {Lon }}$ & $\delta_{\text {Lat }}$ & $\delta_{\text {Ped }}$ \\
\hline$u$ & B1 & B2 & B3 & 0 \\
\hline$w$ & B4 & 0 & 0 & 0 \\
\hline$q$ & B5 & B6 & B7 & B8 \\
\hline$v$ & 0 & 0 & 0 & 0 \\
\hline$p$ & B9 & B10 & B11 & B12 \\
\hline$p$ & B13 & B14 & B15 & B16 \\
\hline$r$ & 0 & 0 & 0 & 0 \\
\hline \hline
\end{tabular}

\subsection{Evaluation of fitness function}


Fitness function is the main criterion of the GA algorithm, as it represents how much the system is optimum and stable. The following equation describes the relation between the fitness function and the performance index as shown in the following equation.

$P I_{2}=\sum_{k=1}^{n}\left(\int_{t=0}^{t_{f}}\left(X_{k}-\hat{X}_{k}\right)^{2} d t\right) g_{k}$

In the above equation $X_{k}$ is the actual system output while $\hat{X}_{k}$ is the predicted output (Inferred State Space), $g_{k}$ is the corresponding attitude weight that depends on the priorities of this attitude. $\mathrm{k}$ is attitude notation corresponding to the states in the state space matrix $A$. Therefore the fitness function could be written as the inverse of the performance index of the inferred state space performance as shown in the following equations. $[9,10,11]$

$$
F\left(A_{1}, A_{2}, \ldots \ldots \ldots A_{26}, B_{1}, B_{2}, \ldots \ldots \ldots B_{18}\right)=\left(P I_{2}\right)^{-1}
$$

\section{$\underline{2.3 \text { Design operators }}$}

Proportion selection operator $\square$ single point crossover operator $\square$ basic bit mutation operator.

\section{4 Parameters of $G A$}

Population size is $M=200$, generation $G=100$, crossover probability $P_{\mathrm{c}}=0.60 \square$ mutation probability $P_{\mathrm{m}}=0.10$ and value code $=10$ bits. Adopting the above steps, after 100 iterations in one round, reasonable values could be obtained. Figure (5) shows that the performance index of the inferred state space performance compared with the actual state space model could reach less than 1000 which is adequately an acceptable number.

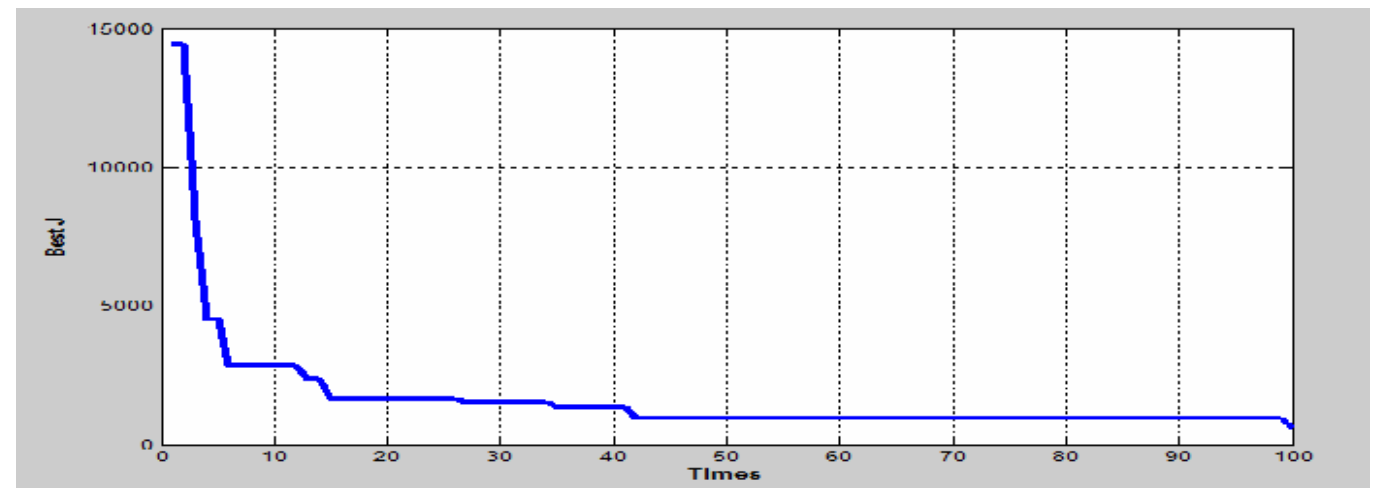

Figure (5): Performance Index for Inferred State Space Compared with the Actual 3-Recurrent Neural Network Based System Identification 
Different neural network structures and training methods were conducted for modeling the nonlinear dynamics of the UAV. The ARX technique proved to be most suitable for this purpose [7]. In the autoregressive neural network model the network retains information about the previous outputs and inputs to predict the next output. This provides equivalent retention capabilities of the dynamics of the UAV by the network. The predicted output of a nonlinear model can be obtained as shown in the following equation. [8]

$$
\begin{gathered}
y(t \theta)=g\left(a_{1} y(t-1)+a_{2} y(t-2)+\cdots+a_{n a} y(t-n a)+b_{1} u(t-1)+\cdots\right. \\
\left.+b_{n b} u(t-n b)\right)
\end{gathered}
$$

Where $\theta$ is the coefficient matrix which gives the influence of past outputs (a........... $a_{\text {na }}$ ) and influence of past inputs $\left(b_{1} \ldots . . b_{n b}\right)$ on each of the subsequent outputs. The nonlinear function is defined by $\mathrm{g}$, the $\mathrm{y}$ and $\mathrm{u}$ terms correspond to past outputs and past inputs respectively. The above equation can be simplified as

$$
\begin{aligned}
& y(t)=g(\theta, \phi(t)) \\
& \theta=\left(a_{1} a_{2} a_{3} a_{n a} \ldots \ldots \ldots b_{1} b_{2} b_{3} b_{n b}\right) \\
& \phi(t)=\left(y(t-1) \ldots \ldots \ldots y\left(t-n_{a}\right) \ldots \ldots . u\left(t-n_{k}\right) \ldots \ldots . u\left(t-n_{k}-n_{b}-1\right)\right)
\end{aligned}
$$

Here $\varphi$ is the matrix of past inputs and outputs called the regressor and it is available from memory. To obtain the coefficients $\theta$, many assumptions and detailed knowledge of the plant are necessary. Hence for a dynamic nonlinear system such as the UAV it may not be feasible. This can be avoided by using black-box methods such as the neural networks. The output of a two layered neural network is given as:

$y_{i}(t)=F_{i}\left(\sum_{j=0}^{l 1} W_{2 i j} G_{j}\left(\sum_{k=1}^{l 2} W_{1 j k} x_{k}(t)+b_{1 j 0}\right)+b_{2 i 0}\right)$

In the above equation $F$ and $G$ are the activation functions, $l_{1}$ and $l_{2}$ the number of neurons in the two layers, $b_{l j 0}$ and $b_{2 j 0}$ are the bias to the two layers and $x_{k}$ is the 
network input. In most of the cases the nonlinearities are best represented by the hyperbolic tangent function as the activation function $G$ and a linear relation $F$. $W_{l j k}$ and $W_{2 i j}$ are the weights from the hidden layer and the output layer respectively. These weights correspond to the $\theta$ matrix. Hence the problem of obtaining the best prediction depends on the network structure adapted and the training method used. Iterative training is performed to minimize an error function using the genetic algorithms. The goal of the training is to obtain the most suitable values of weights for the closest possible prediction output through repetitive iterations. The GA method works on the principle of minimizing the mean squared error between the actual output of the system and the predicted output of the network. The summation of the mean square error of each subsequent attitude $P I_{l}$ (performance index) should be minimized by applying GA.

\subsection{Using binary system coding}

Coding $\square$ using 20-bit binary genes to express $\left(W_{l}, W_{2}, \ldots \ldots \ldots W_{140}\right)$ and $\left(b_{1}\right.$, $\left.b_{2}, \ldots \ldots \ldots b_{13}\right)$. For example $\left(W_{l}\right) \quad$ from $00000000000000000000(0), \quad$ is 1111111111111111111 (1048575). Then string $W_{2} \ldots$. etc. till $b_{13}$ to 3060 -bit binary cluster. $x: 0000110111 \ldots \ldots \ldots . . .11011100010000100010$ expresses a chromosome $\square$ the former 20-bit express $\left(W_{1}\right)$, the second portion express $\left(W_{2}\right)$ and the third one express the $\left(W_{3}\right) \ldots$ etc.

Decoding $\square$ Cut one string of 3060-bit binary string to 306 20-bit binary string $\square$ then converts them to decimal system values $\left(W 1, W 2, \ldots \ldots \ldots W_{140}\right)$ and $\left(b_{1}, b_{2}, \ldots \ldots \ldots b_{13}\right)[3$, 4].

\section{$\underline{3.2 \text { Evaluation of fitness function }}$}

Fitness function is the main criterion of the GA algorithm, as it represents how much the system is optimum and stable. The following equation describes the relation between the fitness function and the performance index as shown in the following equation.

$$
P I_{1}=\sum_{k=1}^{n}\left(\int_{t=0}^{t_{f}}\left(X_{k}-\hat{X}_{k}^{N}\right)^{2} d t\right) g_{k}
$$

In the above equation $X_{k}$ is the actual system output while $\hat{X}_{k}^{N}$ is the predicted output (Recurrent Neural Network), $g_{k}$ is the corresponding attitude weight that depends on the priorities of this attitude. $\mathrm{k}$ is the attitude notation in the state space matrix $A$. therefore 
the fitness function could be written as the inverse of the performance index of the recurrent neural network performance as shown in the following equation.

$F\left(W 1, W 2, \ldots \ldots \ldots W_{140}, b_{1}, b_{2}, \ldots \ldots \ldots b_{13}\right)=\left(P I_{1}\right)^{-1}$

\section{3 Design operators}

Proportion selection operator $\square$ single point crossover operator $\square$ basic bit mutation operator.

\section{4 Parameters of $G A$}

Population size is $M=200$, generation $G=6000$ at least, crossover probability $P_{\mathrm{c}}=0.60 \square$ mutation probability $P_{\mathrm{m}}=0.10$ and cluster length $=20$ bits. Adopting the above steps, consequently after 6000 steps iterations in five rounds a reasonable network performance could be obtained compared with the actual system as shown in Table (7).

Table (7): Five Iterative Rounds for Weight Calculation

\begin{tabular}{|c|c|c|}
\hline Round no. & $\begin{array}{c}\text { Adapted Weight Range ( multiplied by the best } \\
\text { weights from the previous process) }\end{array}$ & $\mathrm{PI}_{1}$ \\
\hline \hline 1 & Predefined Weight Range & 23000 \\
\hline 2 & The Best Weights in round (1) *0.3 & 19500 \\
\hline 3 & The Best Weights in round (2) *0.6 & 12900 \\
\hline 4 & The Best Weights in round (3)*0.9 & 6000 \\
\hline 5 & The Best Weights in round (4) *0.3 & 5100 \\
\hline 6 & The Best Weights in round (5)*0.6 & 4800 \\
\hline
\end{tabular}

In order to optimize the proposed recurrent neural network weights to get a reasonable performance of the network the number of generations must exceed 5000 with 200 population size at least and 20 bits cluster length. This enormous number of iterations would result in a problem during the execution of the genetic algorithm code. Therefore it is recommended to divide this large generation number into 5 or 6 rounds while changing the weight limitations in each round in accordance with the previous best values of the network weights and biases. Performing those rounds mentioned in Table (7) the performance index could be reduced to about 4800 as shown in Figure (6) through Figure (10) which is about five times the final performance index of the inferred state space. 


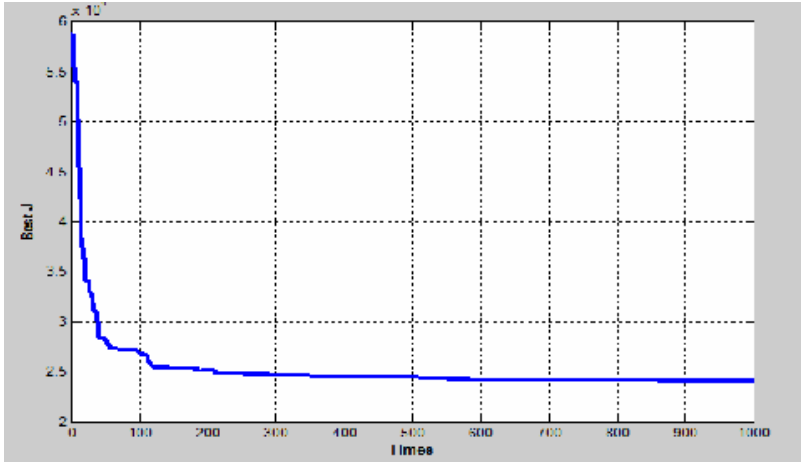

Figure (6): Performance Index in Round (1)

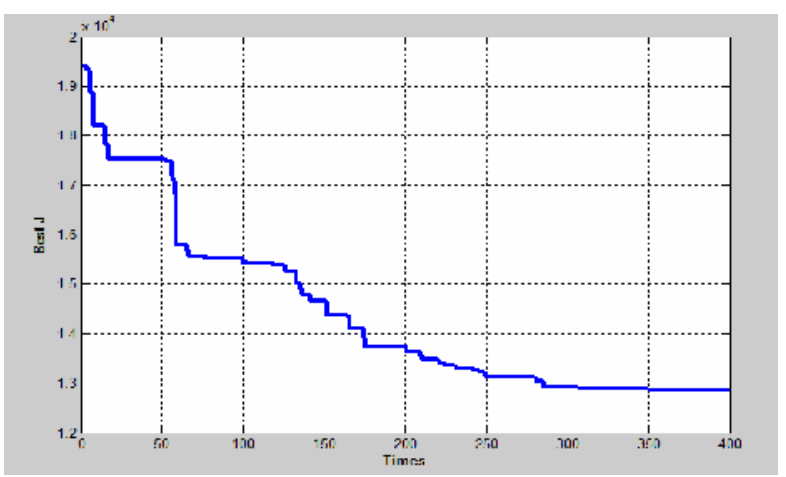

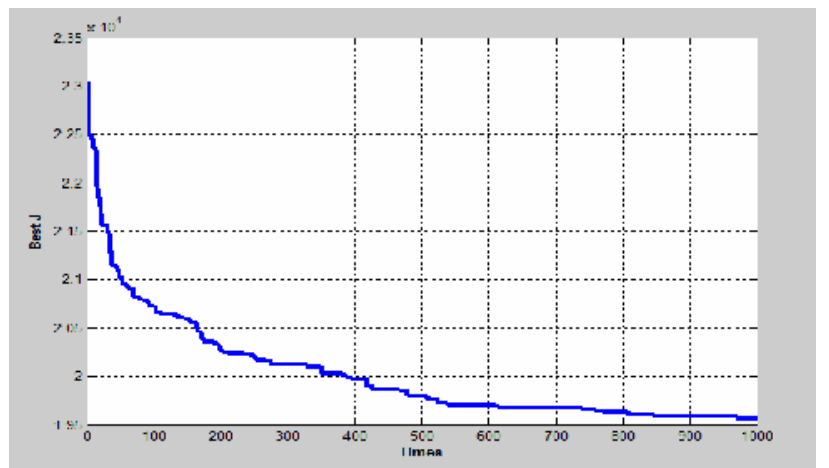

Figure (7): Performance Index in Round (2)

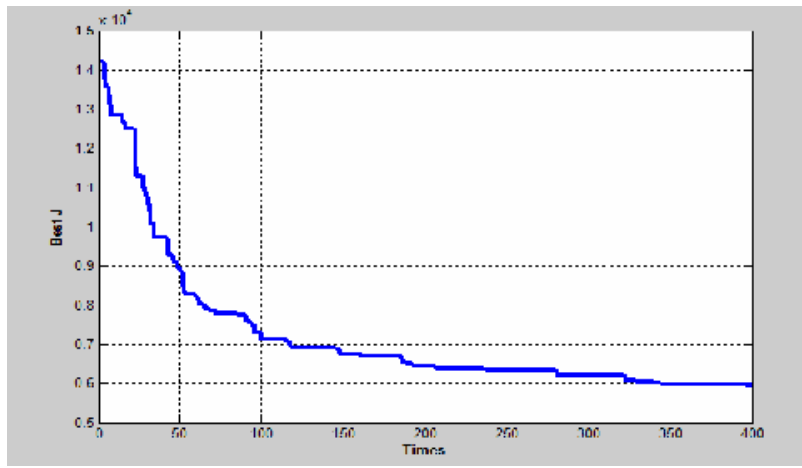

Figure (8): Performance Index in Round (3) Figure (9): Performance Index in Round (4)

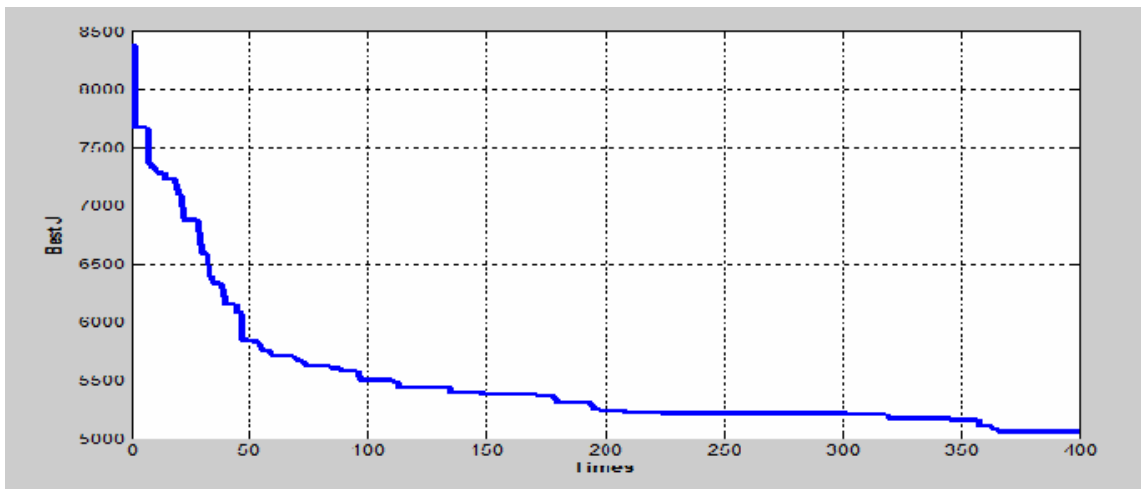

Figure (10): Performance Index in Round (5)

Using iterative rounds with adapting the weights ranges in each round will allow a progressive searching for the global minimum instead of the local one. Figure (11) illustrates the searching process from some local minimum to a next better one until reaching the global minimum or at least the closest value to it in the neighborhood by changing the weights limitations in each round in the GA. 


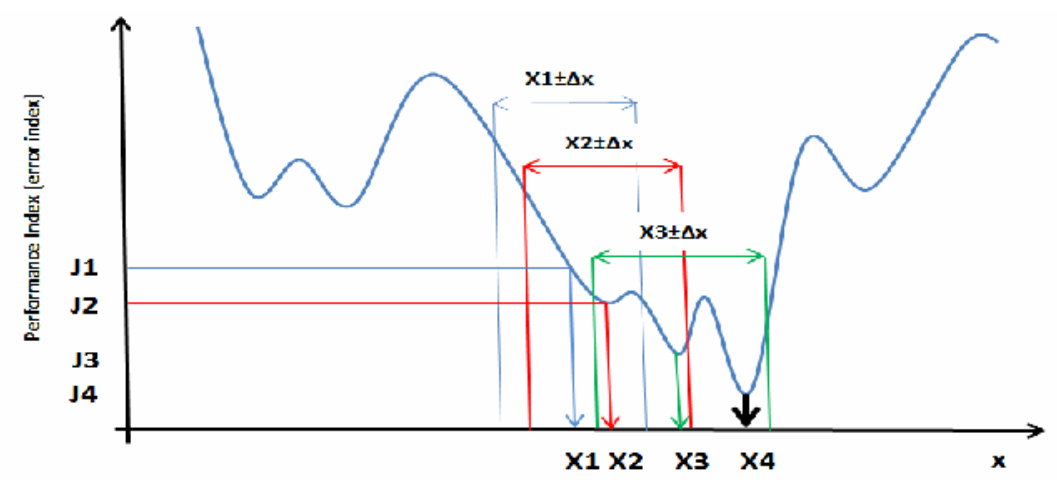

Figure (11): Looking for the Global Minimum by Using Adaptive Genetic Algorithm

\section{System Evaluation}

\section{$\underline{4.1 \text { Evaluation Criteria }}$}

Figure (12) shows the performance index for each proposed systems (inferred state space and recurrent neural network). $\boldsymbol{P I}_{\boldsymbol{I}}$ is the performance index that measures the performance of the proposed recurrent neural network performance compared with the actual model. $\boldsymbol{P I}_{2}$ is the performance index that measures the proposed inferred state space performance compared with the actual state space model. Both systems were examined and measured using an adequate random input from an arbitrary signal generator. A Simulink model was implemented to obtain these performance indexes with respect to time as shown in Figure (13).

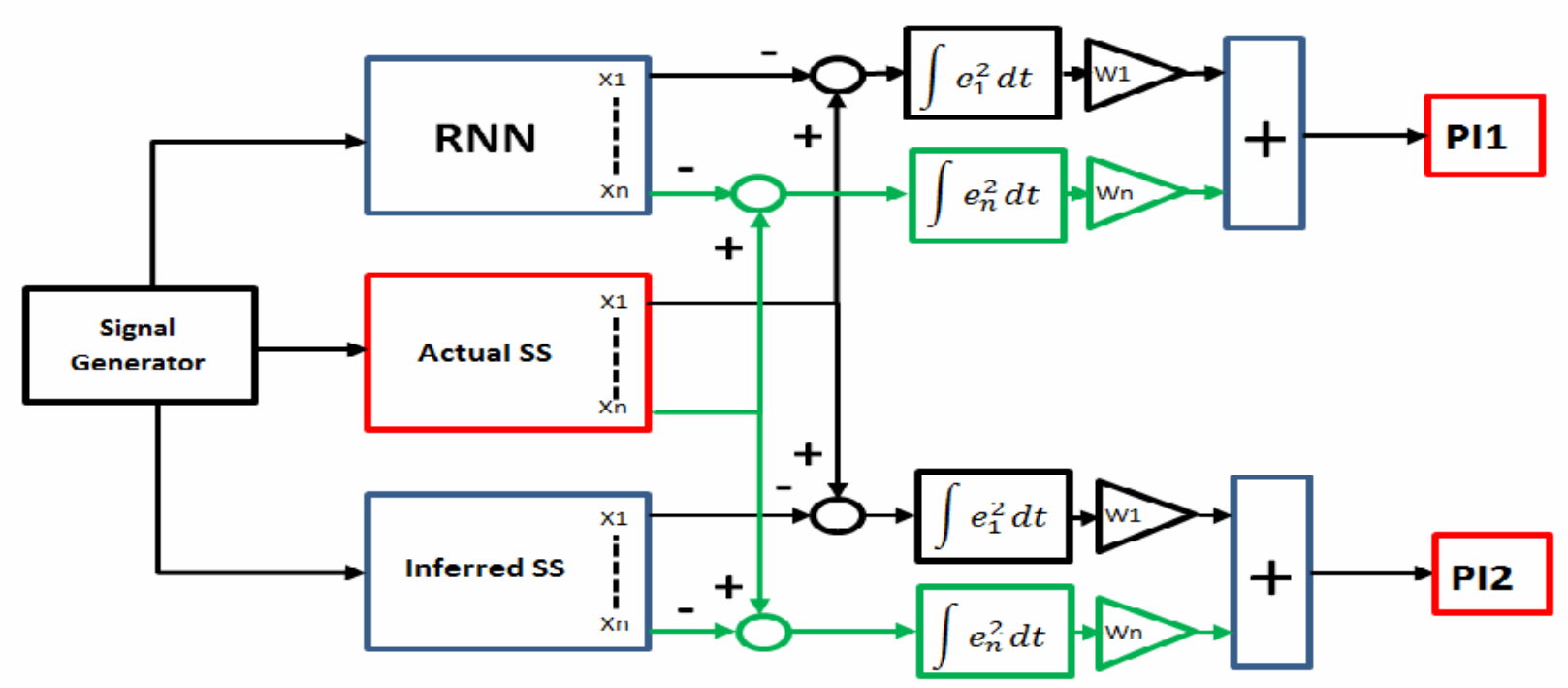

Figure (12): Performance Index for both NN and SS Based System Identifications 


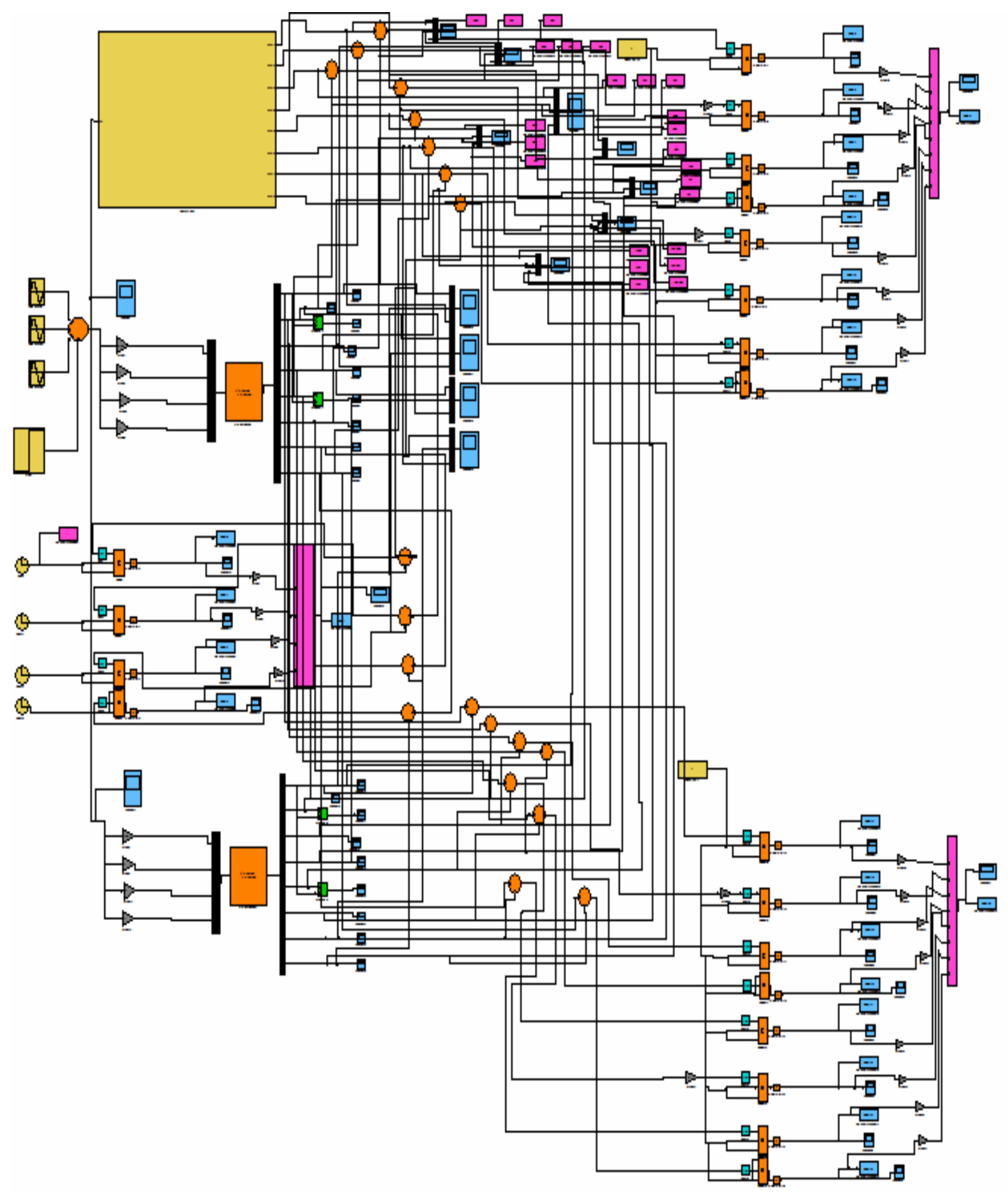

Figure (13): Simulink Model for Calculating the Performance Indexes for both NN and SS models 
Figure (14) shows the performance index for inferred state space and neural network based system identifications describing the sharp increase in the performance index of the neural network based system identification relative to the performance index of the inferred state space system identification. Therefore the inferred state space based system identification is the closest proposed model that can simulate the actual performance of the nonlinear model with an acceptable error.

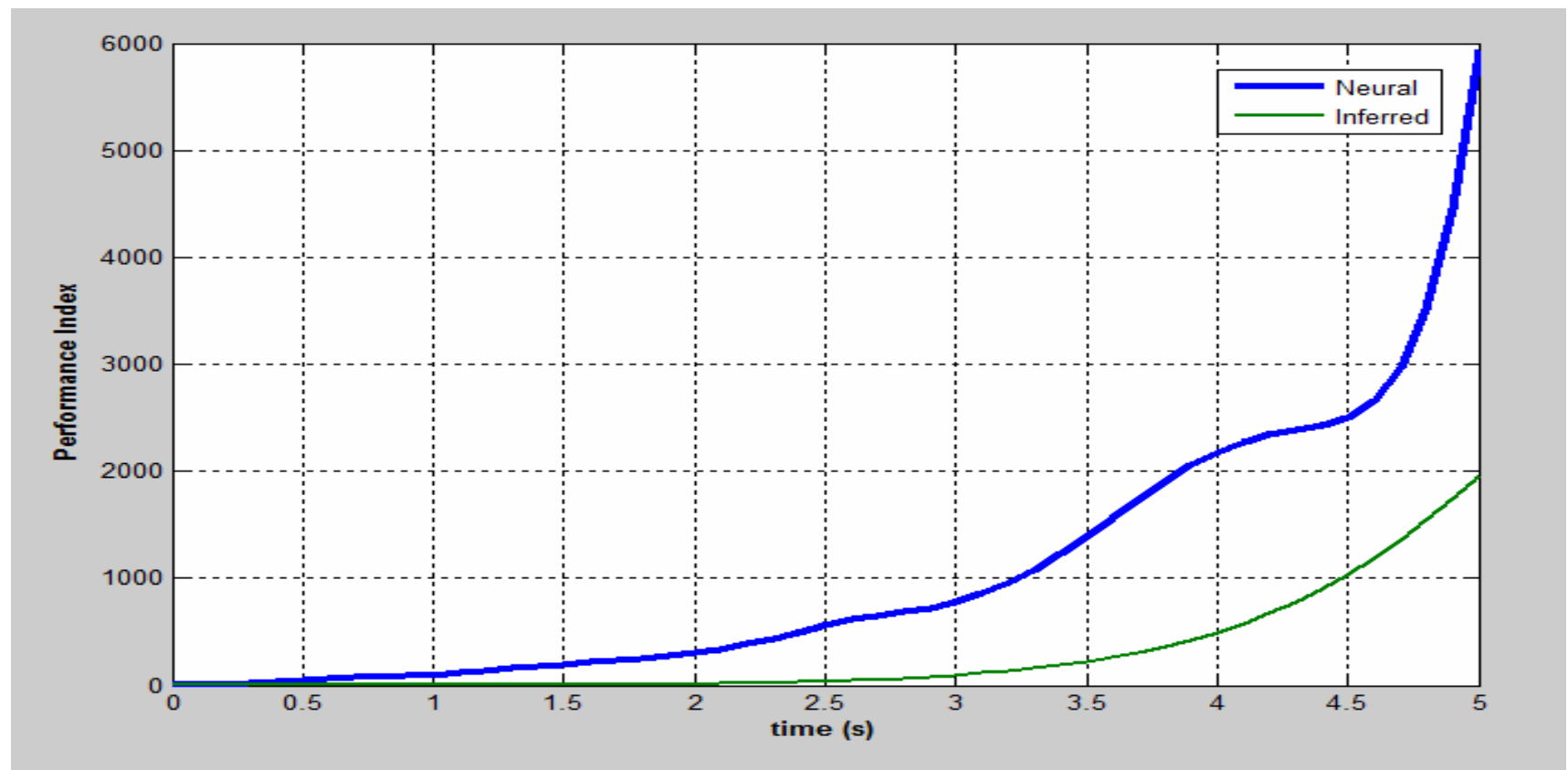

Figure (14): Performance Indexes for both NN and SS models with respect to the Actual Model

\subsection{System Performance}

Figure (15) to Figure (22) illustrate the different attitudes for both inferred state space and neural network system identifications for arbitrary combined sinusoidal input signals. These figures show an acceptable performance of the inferred state space based system identification compared with the neural network based system identification with respect to the actual model. Figure (23) to Figure (26) present the dynamic behavior of both inferred and actual state space of unmanned helicopter model integrated with PID controller. The dynamic response shows that the inferred state space model has an acceptable performance according to a unit step command relative to the actual model. 


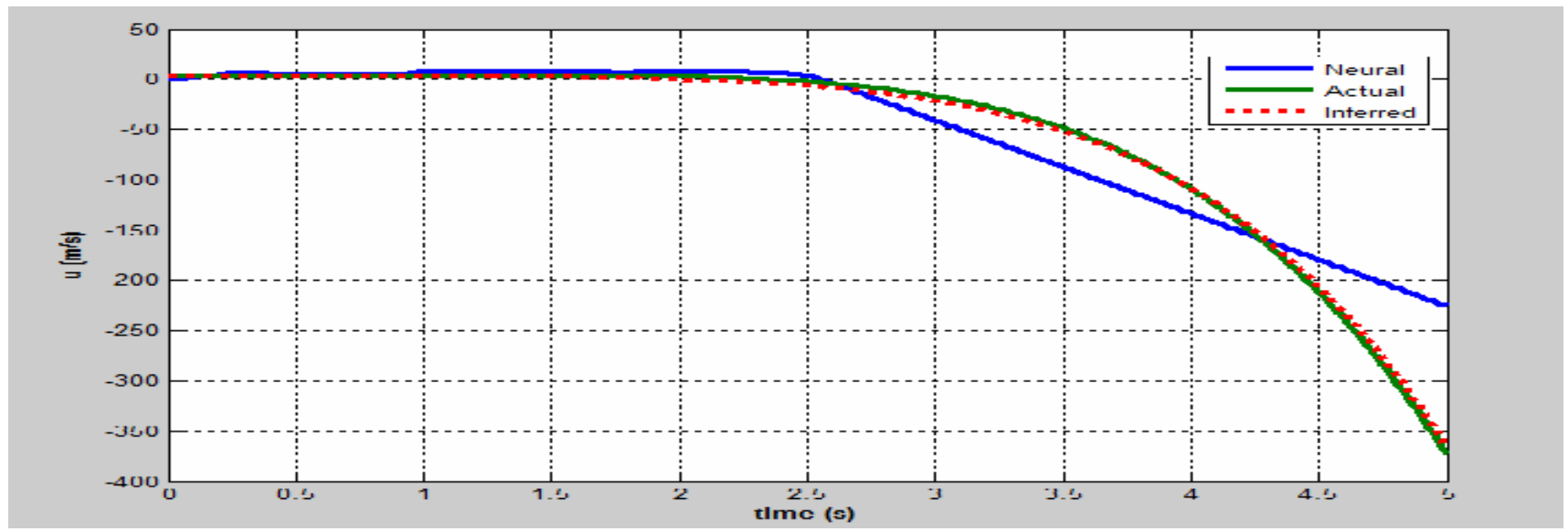

Figure (15): Forward Speed for Neural Network, Actual and Inferred State Space models

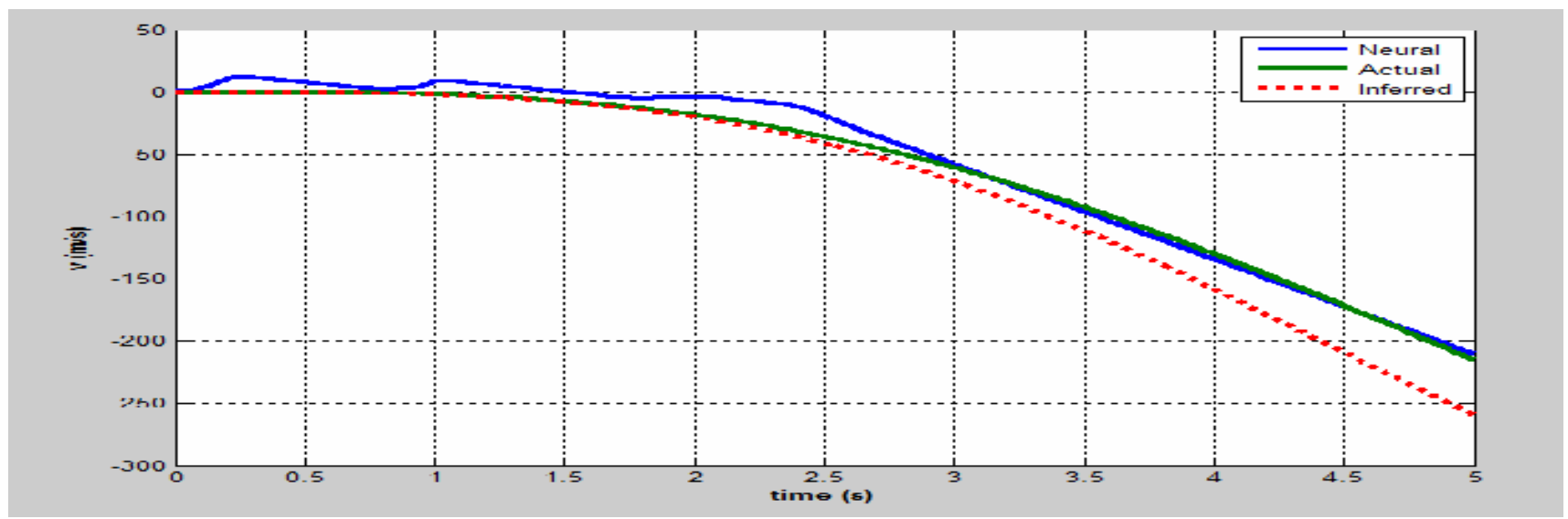

Figure (16): Side Speed for Neural Network, Actual and Inferred State Space models

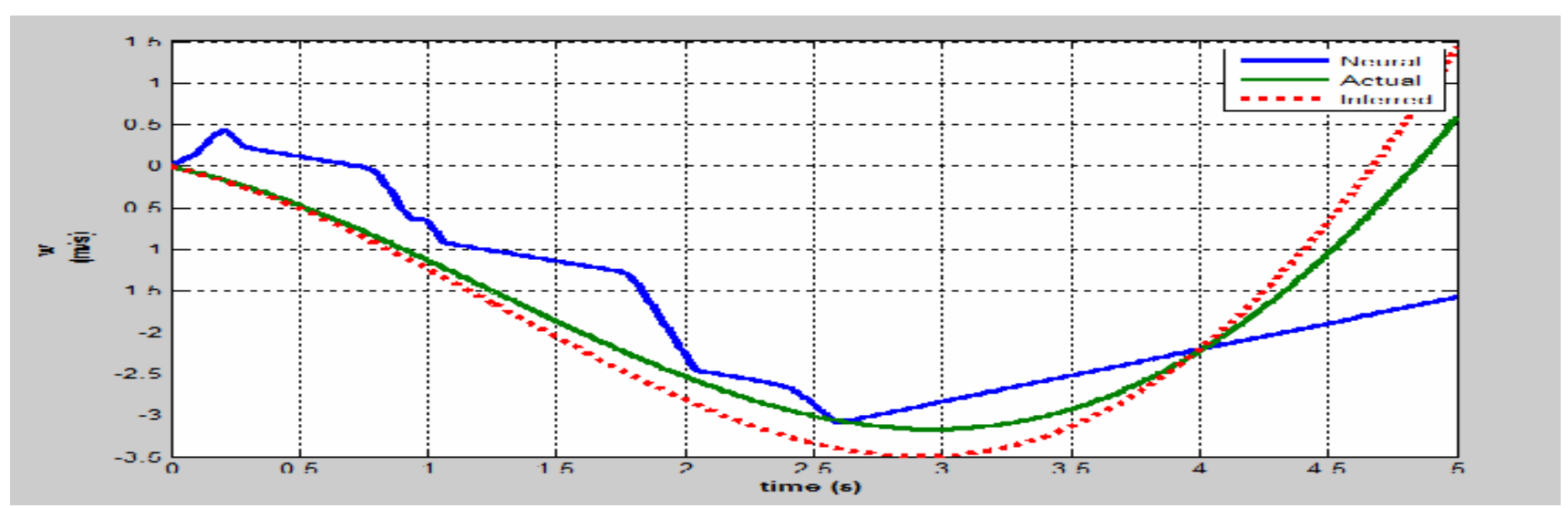

Figure (17): Vertical Speed for Neural Network, Actual and Inferred State Space Models 


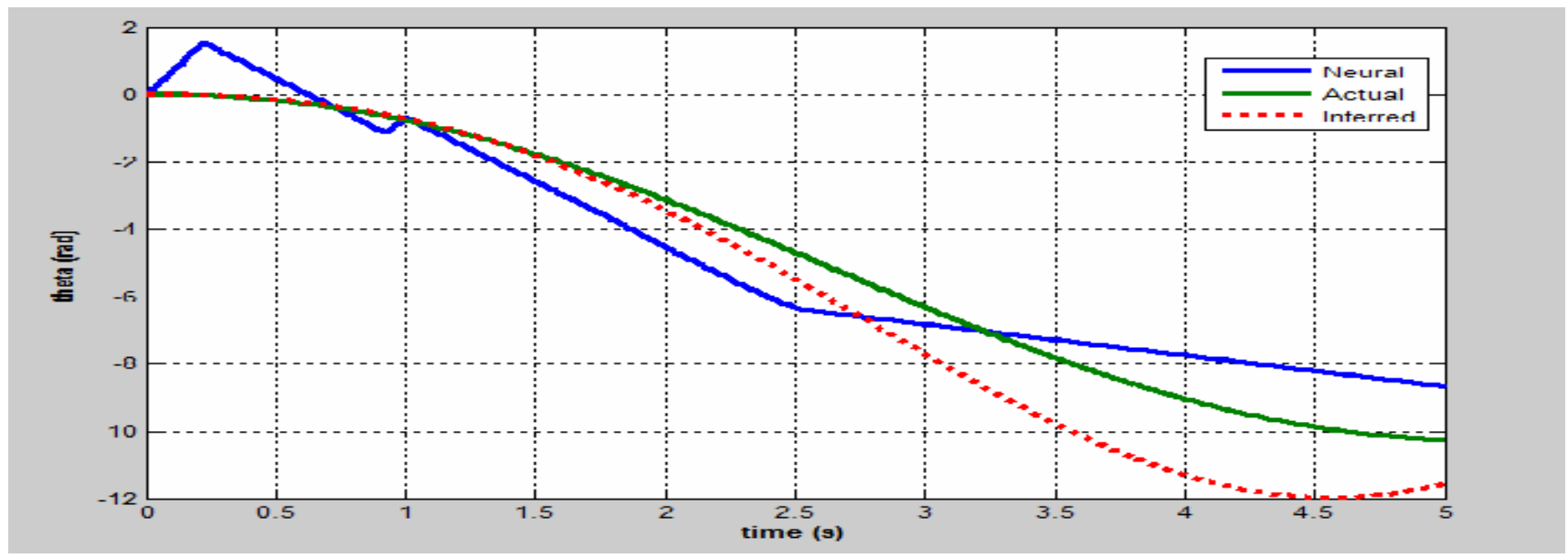

Figure (18): Elevation Angle for Neural Network, Actual and Inferred State Space models

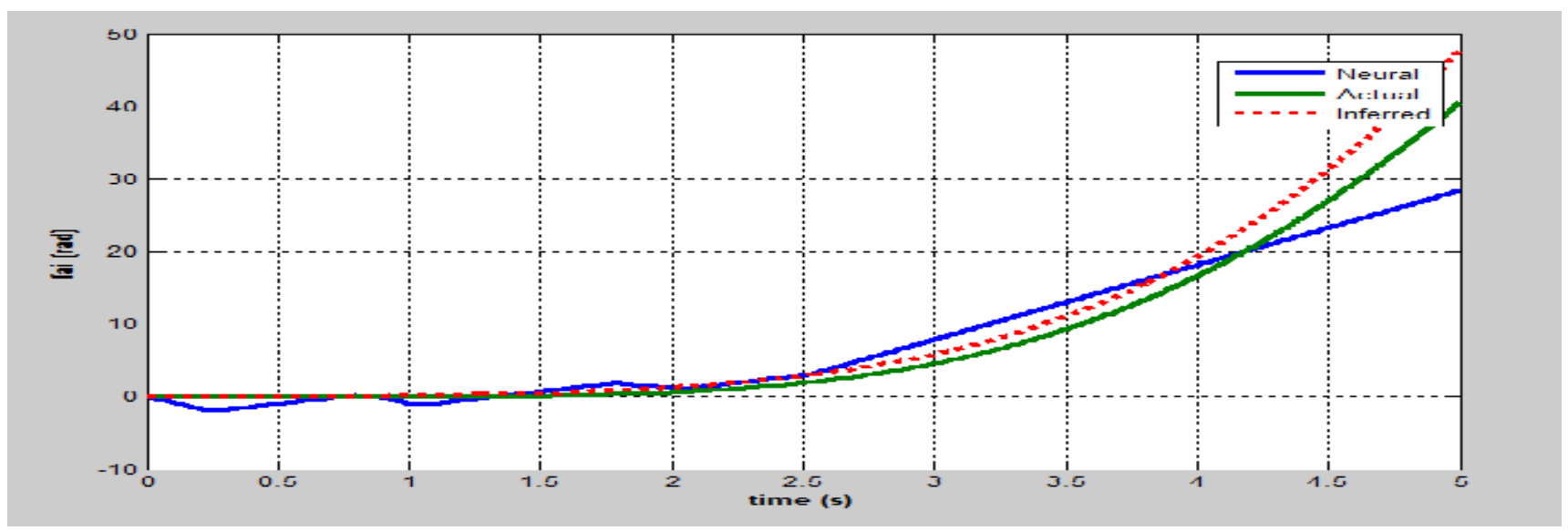

Figure (19): Bank Angle for Neural Network, Actual and Inferred State Space models

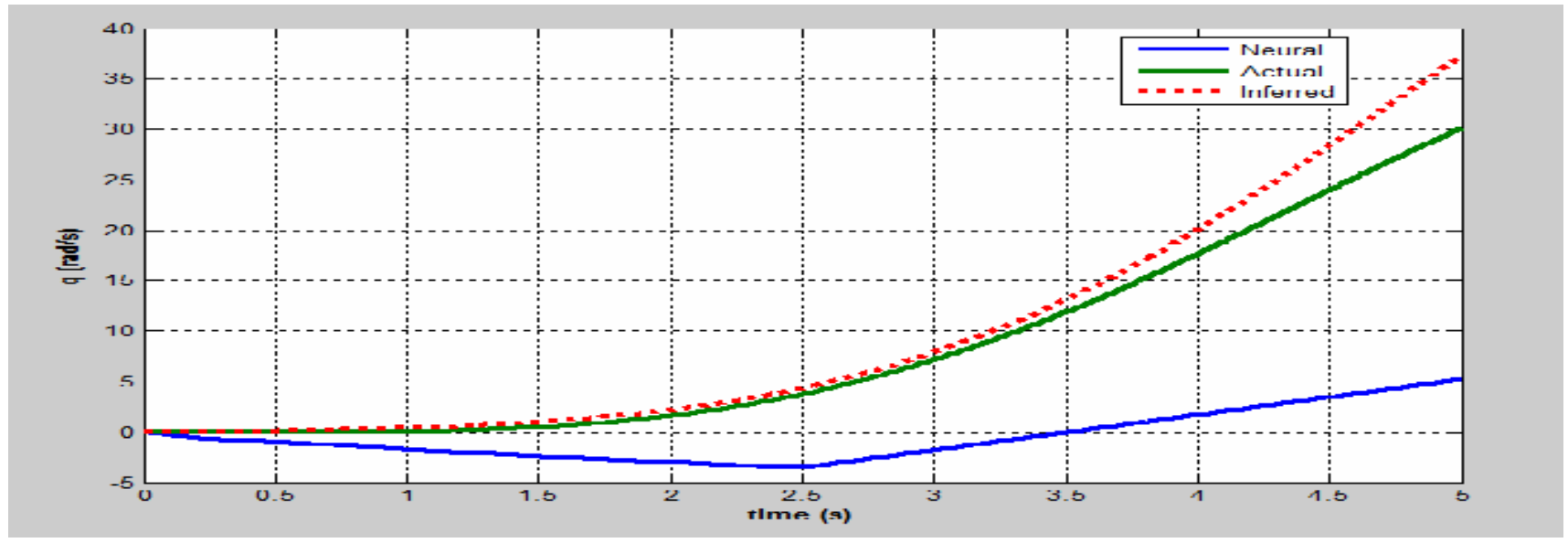

Figure (20): Pitch Rate Speed for Neural Network, Actual and Inferred State Space models 


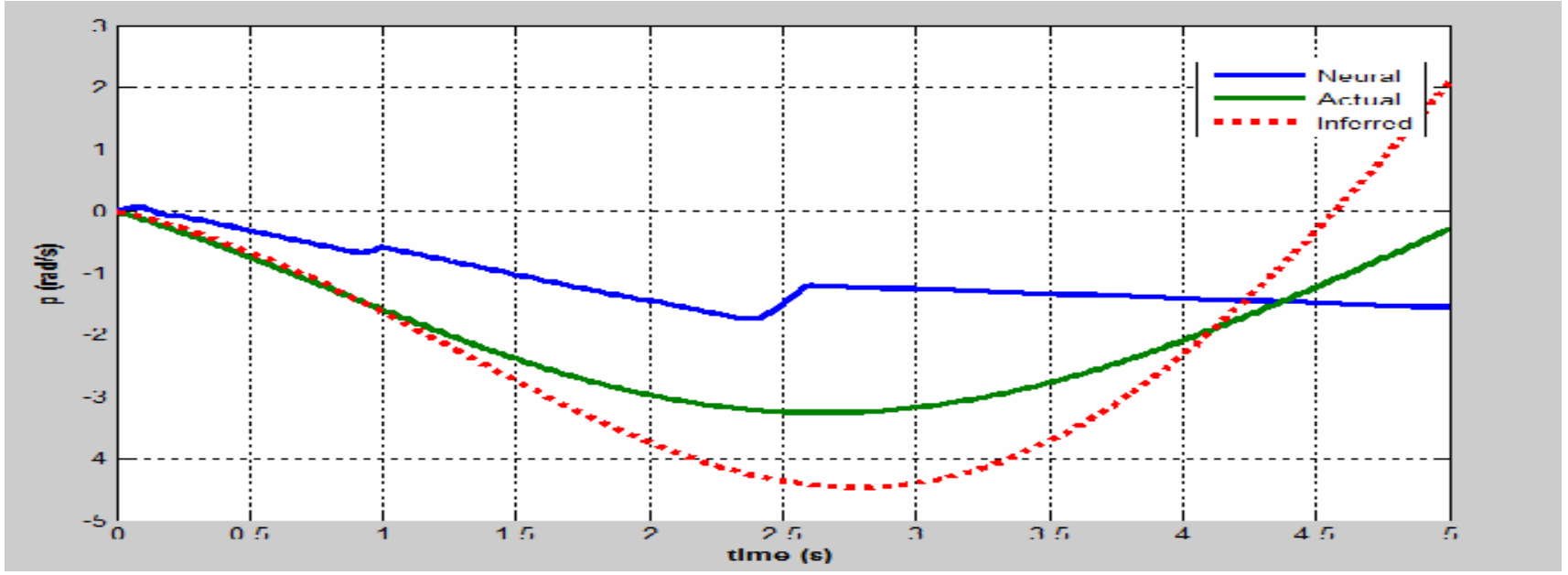

Figure (21): Roll Rate for Neural Network, Actual and Inferred State Space models

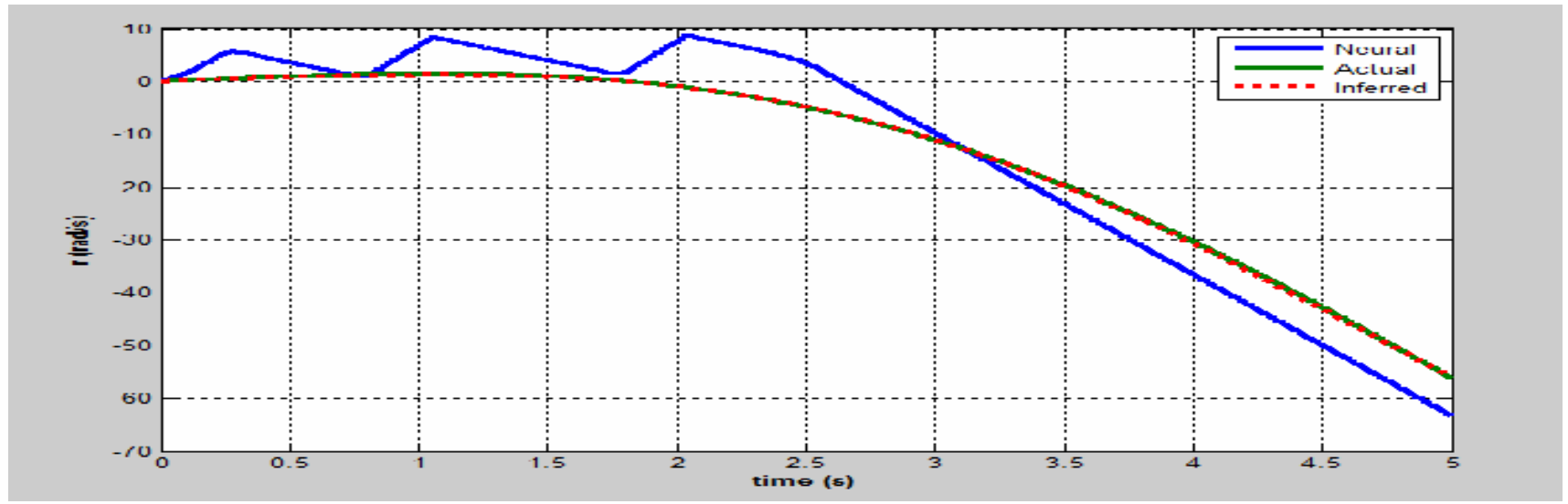

Figure (22): Yaw Rate for Neural Network, Actual and Inferred State Space models

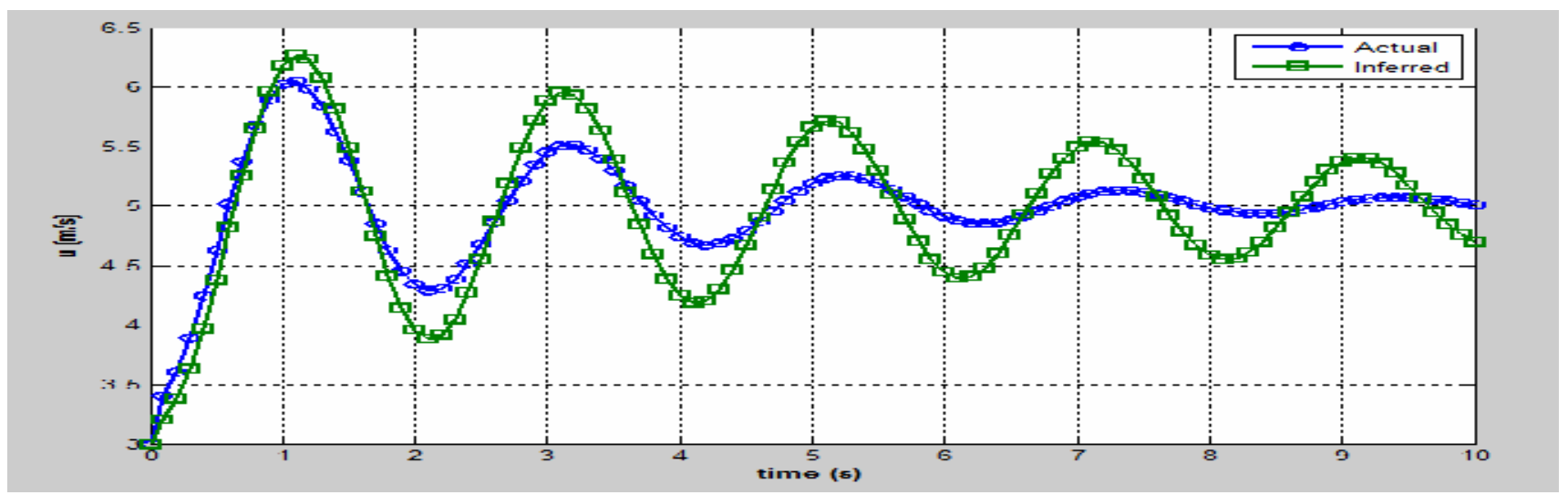

Figure (23): Forward Speed Performance due to Unit Step Command Using PID Controller 


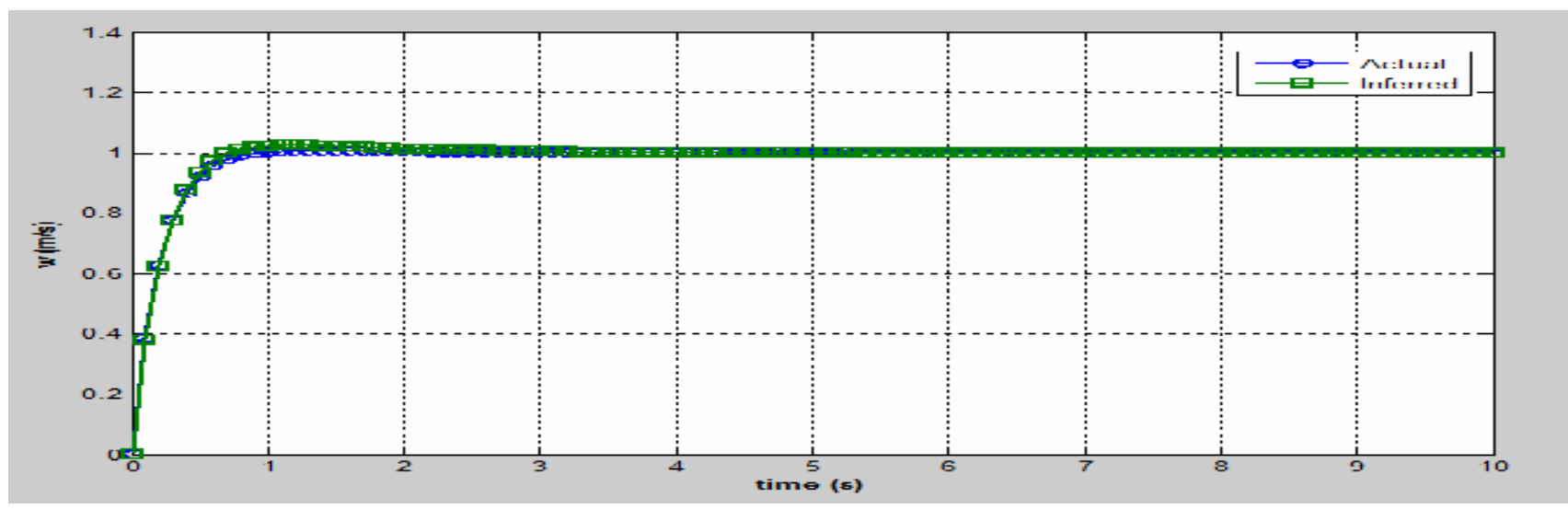

Figure (24): Vertical Speed Performance due to Unit Step Command Using PID Controller

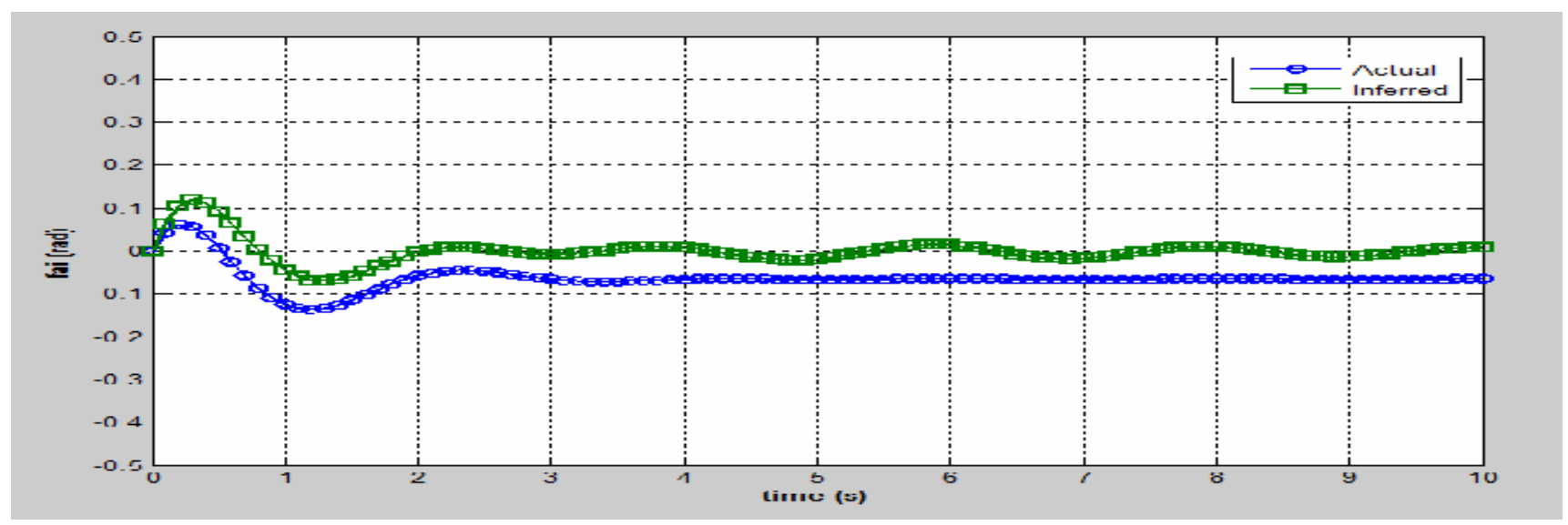

Figure (25): Bank Angle Performance due to Unit Step Command Using PID Controller

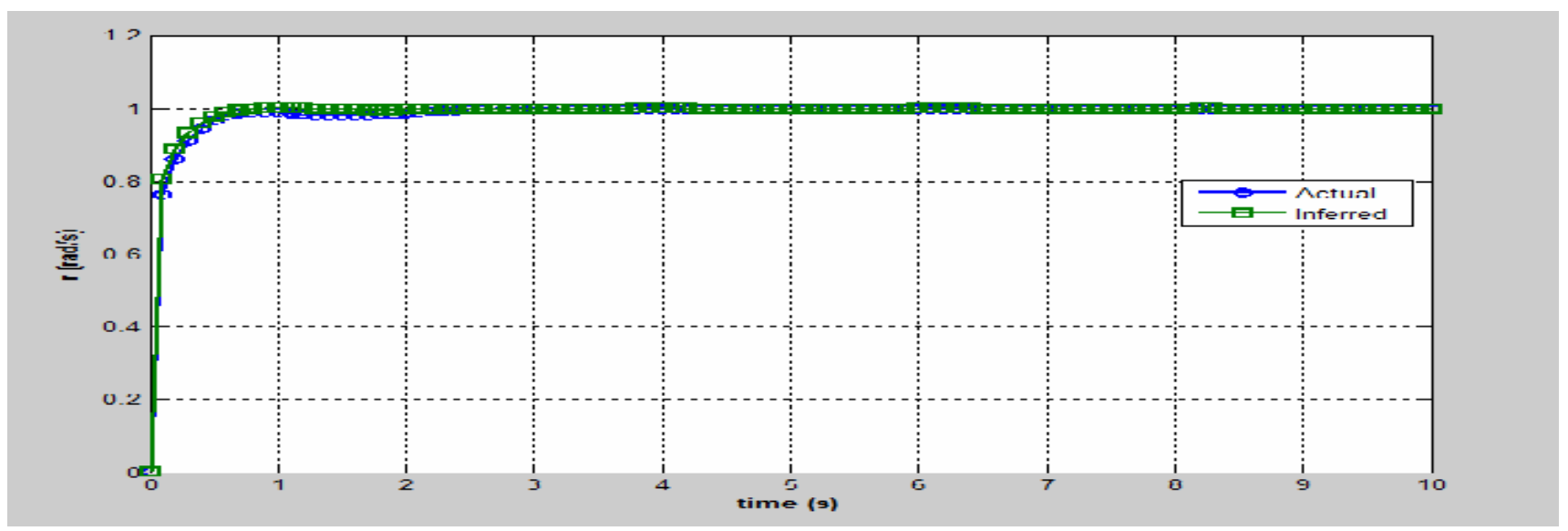

Figure (26): Yaw Rate Performance due to Unit Step Command Using PID Controller 
Figure (27) shows the dynamic poles for the proposed system identification (inferred state space) and for the actual one. It is one of the methods used to evaluate the proposed system identification (state space based system identification) relative to the actual one. For precise measuring technique it is recommended to calculate the relative margin between the proposed system and the actual model poles instead of calculating the absolute margin. This method would present a better expression for the degree of closeness of the proposed model to the actual one as shown in the following Figure (27).

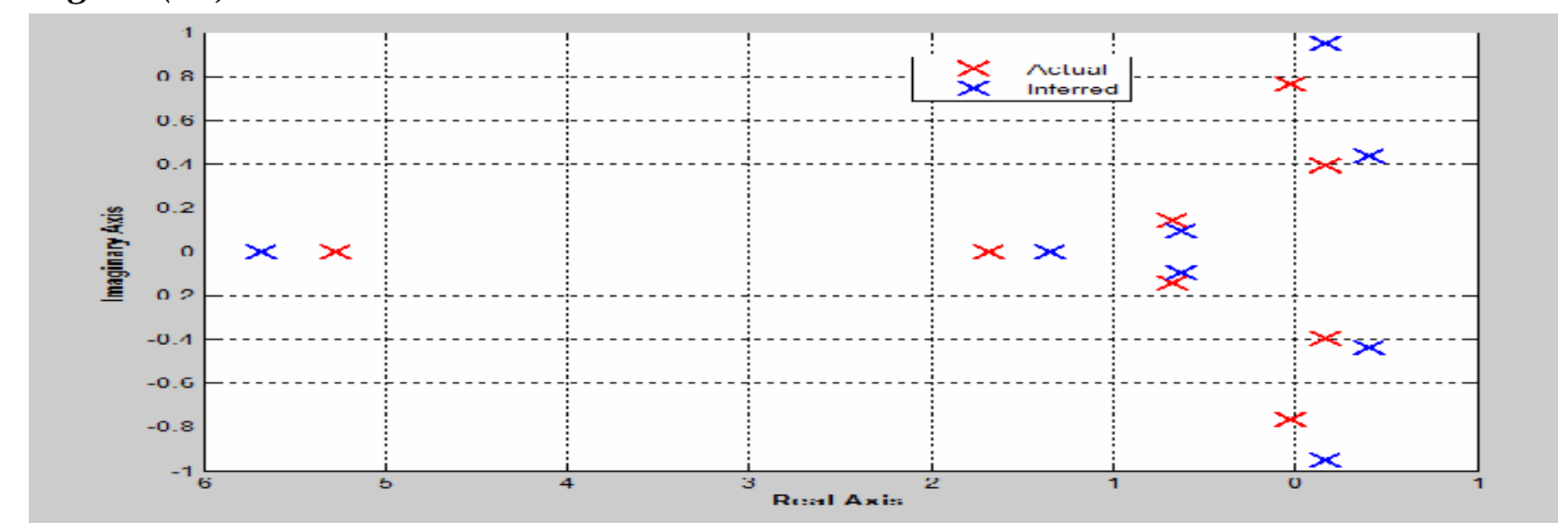

Figure (27): Actual and Inferred State Space Poles

\section{$\underline{\text { 5. Conclusion }}$}

In this work it is obvious to conclude that the system identification based on an inferred state space model gives better performance than the neural network based model according to the performance index for each one. Moreover the computation time to obtain the inferred state space elements is lesser than to obtain the neural network weights. The computation time needed to calculate the recurrent neural network weights is considerably high due to the large number of the weights and biases to establish multi-layer recurrent neural network RNN. As the second objective in this work is to minimize the performance index of the inferred state space ISS, there might be some different combinations give approximately the same behavior of the real system, so it is recommended to increase the time interval of calculating the performance index besides increasing the number of the generations of the genetic algorithm with a reasonable number of cluster bits to reach to the closest combination of the inferred state space elements to the actual state space elements that could simulate the true model with acceptable performance. 


\section{References}

[1] Shamsudin S. S., "The Development of Autopilot System for an Unmanned Aerial Vehicle (UAV) Helicopter Model", "University of Technology, M.Sc." August, 2007, pp. 1-147.

[2] Hosny A. M., Chao H., “ Development of Fuzzy Logic LQR Control Integration for Aerial Refueling Autopilot", " Proceedings of the $12^{\text {th }}$ International Conference on Aerospace Sciences and Aviation Technology, ASAT-12," May 29-31, 2007.

[3] Hosny A. M., Chao H., "Fuzzy Logic Controller Tuning Via Adaptive Genetic Algorithm Applied to Aircraft Longitudinal Motion", " Proceedings of the $12^{\text {th }}$ International Conference on Aerospace Sciences and Aviation Technology, ASAT12," May 29-31, 2007.

[4] Goldberg, D., Genetic Algorithms in Search, Optimization \& Machine Learning, ${ }^{\text {st }}$. ed., Vol. 1, Addison Wesley Longman, 1989.

[5] Flying Qualities of Aeronautical Design Standard for military helicopter (ADS33C) (US Army Aviation Systems Command, 1989).

[6] Hosny A. M., Hosny M. M. and Ez El-Deen H., "Development of a Customized Autopilot for Unmanned Helicopter Model Using Genetic Algorithm via the Application of Different Guidance Strategies" International Conference on Aerospace Sciences and Aviation Technology, ASAT-14," May 24-26, 2011.

[7] Lennart Ljung, "System Identification - Theory for the User", Prentice Hall, 2nd edition, 1999, Prentice Hall, 2nd edition, 1999.

[8] Vishwas Puttige and Sreenatha Anavatti. "Real-time Neural Network Based Online Identification Technique for a UAV Platform", In International Conference on Computational Intelligence for Modeling Control Application, page 92, Washington, DC, USA, 2006, IEEE Computer Society. 